\title{
Effect of Variable Viscosity on Vortex Instability of Non-Darcy Mixed Convection Boundary Layer Flow Adjacent to a Nonisothermal Horizontal Surface in a Porous Medium
}

\author{
A. M. Elaiw,,$^{1,2}$ A. A. Bakr, ${ }^{3}$ M. A. Alghamdi, ${ }^{1}$ and F. S. Ibrahim ${ }^{4}$ \\ ${ }^{1}$ Department of Mathematics, Faculty of Science, King Abdulaziz University, P.O. Box 80203, \\ Jeddah 21589, Saudi Arabia \\ ${ }^{2}$ Department of Mathematics, Faculty of Science, Al-Azhar University, Assiut 71511, Egypt \\ ${ }^{3}$ Department of Mathematics, Faculty of Science for Girls, King Khalid University, Abha, Saudi Arabia \\ ${ }^{4}$ Department of Mathematics, University Collage in Makkah, Umm-Alqura University, Saudi Arabia
}

Correspondence should be addressed to A. M. Elaiw, a_m_elaiw@yahoo.com

Received 6 July 2011; Accepted 11 November 2011

Academic Editor: Muhammad R. Hajj

Copyright ( 2012 A. M. Elaiw et al. This is an open access article distributed under the Creative Commons Attribution License, which permits unrestricted use, distribution, and reproduction in any medium, provided the original work is properly cited.

\begin{abstract}
We study the effect of variable viscosity on the flow and vortex instability for non-Darcy mixed convection boundary layer flow on a nonisothermal horizontal plat surface in a saturated porous medium. The variation of viscosity is expressed as an exponential function of temperature. The analysis of the disturbance flow is based on linear stability theory. The base flow equations and the resulting eigenvalue problem are solved using finite difference schemes. It is found that the variable viscosity effect enhances the heat transfer rate and destabilizes the flow for liquid heating, while the opposite trend is true for gas heating.
\end{abstract}

\section{Introduction}

The study of convective heat transfer from surfaces embedded in porous media has received considerable attention in the literature. The interest for such studies is motivated by several thermal engineering applications, such as storage of radioactive nuclear waste materials transfer, separation processes in chemical industries, filtration, transpiration cooling, transport processes in aquifers, ground water pollution, and thermal insulation. The presence of a buoyancy force component in the direction normal to the surface leads to vortex instability of the flow. The problem of the vortex mode of instability in mixed or natural convection flow over a horizontal or an inclined heated plate in a saturated porous medium has recently received considerable attention (see [1-10]). These studies used either Darcy 
model [1-6], or non-Darcy model [7-10]. A comprehensive literature survey on this subject can be found in the recent book by Nield and Bejan [11].

All the above studies dealt with constant viscosity. The fundamental analysis of convection through porous media with temperature-dependent viscosity is driven by several contemporary engineering applications from cooling of electronic devices to porous journal bearings and is important for studying the variations in constitutive property. The effect of variable viscosity for convective heat transfer through porous media is studied by several investigators [12-20]. The effect of variation of viscosity to study the instability of flow and temperature fields is discussed by Kassoy and Zebib [12] and Gray et al. [13]. Ling and Dybbs [14] presented a very interesting theoretical investigation of temperature-dependent fluid viscosity influence on the forced convection flow through a semi-infinite porous medium bounded by an isothermal flat plate. Lai and Kulacki [15] considered the variable viscosity effect for mixed convection flow along a vertical plate embedded in saturated porous medium. The effect of variable viscosity on combined heat and mass transfer in mixed convection about a wedge embedded in a saturated porous media for the case of uniform heat mass flux (UHF/UMF) is analyzed by Hassanien et al. [17]. The effect of variable viscosity on non-Darcy, free or mixed convection flow on a horizontal surface in a saturated porous medium, is studied by Kumari [16]. The effect of the temperature-dependent viscosity on mixed convection boundary layer assisting and opposing flows over a vertical surface embedded in a porous medium is investigated by Chin et al. [18]. Seddeek [19] studied the effects of magnetic field and variable viscosity on forced non-Darcy flow about a flat plate with variable wall temperature in porous media in the presence of suction and blowing. In [17], the linear variation of fluid viscosity is assumed while, in [15] and [16], the viscosity of the fluid is assumed to vary as an inverse linear function of temperature. In [20], the variation of viscosity with temperature is represented by an exponential function.

The effect of variable viscosity on vortex instability of a horizontal free convection boundary layer flow in a saturated porous medium for an isothermal surface was studied by Jang and Leu [20]. However, the variable viscosity behavior on the flow and vortex instability of non-Darcy mixed convection boundary layer flow over a nonisothermal horizontal plate dose not seem to have been investigated. This motivated the present investigation.

The present study examines in details the effects of temperature-dependent viscosity on the flow and vortex instability of non-Darcy mixed convection boundary layer flow adjacent to a heated horizontal surface embedded in a porous medium with variable wall temperature. The variation of viscosity with temperature is represented by an exponential function, which is more accurate than a linear function for large temperature differences. The transformed boundary layer equations, which are given by using nonsimilar solution approach, are solved by means of a finite difference method. The analysis of the disturbance flow is based on linear stability theory. The disturbance quantities are assumed to be in the form of a stationary vortex roll that is periodic in the spanwise direction, with its amplitude function depending primarily on the normal coordinate and weakly on the streamwise coordinate. The resulting eigenvalue problems are solved by using a finite difference scheme.

\section{Analysis}

\subsection{The Main Flow}

We considered a semi-infinite nonisothermal horizontal surface $\left(T_{w}\right)$ embedded in a porous medium $\left(T_{\infty}\right)$, where $x$ represents the distance along the plate from its leading edge, and $y$ 
the distance normal to the surface. The wall temperature is assumed to be a power function of $x$, that is, $T_{w}=T_{\infty}+A x^{\lambda}$, where $A$ is a constant, and $\lambda$ is the parameter representing the variation of the wall temperature. In order to study transport through high porosity media, the original Darcy model is improved by including inertia. For the mathematical analysis of the problem, we assume that (i) local thermal equilibrium exists between the fluid and the solid phase, (ii) the physical properties are considered to be constant, except for the viscosity $\mu$ and the density $\rho$ in the buoyancy force, (iii) we consider the non-Darcy model given by Ergun [21], and (iv) the Boussinesq approximation is valid. With these assumptions, the governing equations are given by

$$
\begin{gathered}
\frac{\partial u}{\partial x}+\frac{\partial v}{\partial y}=0 \\
u+\frac{K^{*}}{v} u^{2}=-\frac{K}{\mu} \frac{\partial P}{\partial x} \\
v+\frac{K^{*}}{v} v^{2}=-\frac{K}{\mu}\left(\frac{\partial P}{\partial y}+\rho g\right), \\
u \frac{\partial T}{\partial x}+v \frac{\partial T}{\partial y}=\alpha \frac{\partial^{2} T}{\partial y^{2}}
\end{gathered}
$$

where $\rho=\rho_{\infty}\left[1-\beta\left(T-T_{\infty}\right)\right]$ is the fluid density, $u$ and $v$ are the velocities in the $x$ and $y$ directions, respectively, $P$ is the pressure, $T$ is the temperature, $\mu$ is the dynamic viscosity, $K$ is the permeability of the porous medium, $g$ is the gravitational acceleration, $\beta$ is the thermal expansion coefficient of the fluid, and $\alpha$ represents the equivalent thermal diffusivity. Note that the second term on the left-hand side of (2.2) and (2.3) represents the inertia force, where $K^{*}$ is the inertia coefficient in Ergun model. As $K^{*}=0,(2.2)$ and (2.3) reduce to Darcy model.

The viscosity $\mu$ of the fluid is assumed to vary with temperature according to an exponential function

$$
\mu=\mu_{\infty} e^{A_{1}\left(\left(T-T_{\infty}\right) /\left(T_{w}-T_{\infty}\right)\right)},
$$

where $\mu_{\infty}$ is the absolute viscosity at ambient temperature, and $A_{1}$ is constant adopted from the least square fitting for a particular fluid. Formula (2.5) is a generalization of that used in [20], where the wall temperature is taken to be constant.

The pressure terms appearing in (2.2) and (2.3) can be eliminated through crossdifferentiation. The boundary layer assumption yields $\partial / \partial x \ll \partial / \partial y$ and $v \ll u$. With $\psi$ being a stream function such that $u=\partial \psi / \partial y$ and $v=-\partial \psi / \partial x$, the equations (2.1)-(2.4) become

$$
\begin{gathered}
\left(\mu+2 \rho_{\infty} K^{*} \frac{\partial \psi}{\partial y}\right) \frac{\partial^{2} \psi}{\partial y^{2}}+u \frac{\partial \mu}{\partial y}=-K \rho_{\infty} g \beta \frac{\partial T}{\partial x} \\
\frac{\partial \psi}{\partial y} \frac{\partial T}{\partial x}-\frac{\partial \psi}{\partial x} \frac{\partial T}{\partial y}=\alpha \frac{\partial^{2} T}{\partial y^{2}} .
\end{gathered}
$$


The boundary conditions are defined as follows:

$$
\begin{gathered}
v(x, 0)=-\frac{\partial \psi}{\partial x}=0, \quad T(x, 0)=T_{w}=T_{\infty}+A x^{\lambda}, \\
u(x, \infty)=B x^{m} \quad T(x, \infty)=T_{\infty} .
\end{gathered}
$$

Here $m=0$ for assisting flow over a horizontal flat plate at zero incident and $m=1$ for stagnation point flow about a horizontal surface.

On applying the following transformations:

$$
\begin{gathered}
\eta(x, y)=\frac{y}{x} \mathrm{Pe}_{x}^{1 / 2}, \quad f(\xi, \eta)=\frac{\psi(x, y)}{\alpha \mathrm{Pe}_{x}^{1 / 2}} \\
\theta(\xi, \eta)=\frac{T-T_{\infty}}{T_{w}-T_{\infty}}, \quad \xi=\left(\frac{x}{d}\right)^{(2 \lambda-3 m-1) / 2}
\end{gathered}
$$

into (2.5)-(2.7) lead to the following:

$$
\begin{gathered}
\frac{\mu}{\mu_{\infty}}=e^{A_{1}\left(\left(T-T_{\infty}\right) /\left(T_{w}-T_{\infty}\right)\right)}=\left(\mu^{*}\right)^{\theta} \\
\left(1+2 \operatorname{ErPe}_{d} \xi^{2 m /(2 \lambda-3 m-1)}\left(\mu^{*}\right)^{-\theta} f^{\prime}\right) f^{\prime \prime} \\
=-\left(\ln \mu^{*}\right) f^{\prime} \theta^{\prime}-M \xi\left(\mu^{*}\right)^{-\theta}\left(\lambda \theta+\frac{m-1}{2} \eta \theta^{\prime}+\frac{2 \lambda-3 m-1}{2} \xi \frac{\partial \theta}{\partial \xi}\right), \\
\theta^{\prime \prime}=\left(\lambda \theta+\frac{2 \lambda-3 m-1}{2} \xi \frac{\partial \theta}{\partial \xi}\right) f^{\prime}-\left(\frac{m+1}{2} f+\frac{2 \lambda-3 m-1}{2} \xi \frac{\partial f}{\partial \xi}\right) \theta^{\prime},
\end{gathered}
$$

where $\mu^{*}=\mu_{w} / \mu_{\infty}=e^{A_{1}}$ is the wall to ambient viscosity ratio parameter, $\mathrm{Pe}_{x}=u_{\infty} x / \alpha$ is the local Peclet number, $M=R a_{d} / \mathrm{Pe}_{d}^{3 / 2}$ is the mixed convection parameter, $\mathrm{Pe}_{d}=u_{\infty} d / \alpha$ is the Peclet number based on the pore diameter, and $\mathrm{Er}=K^{*} \alpha / d v_{\infty}$ is the Ergun number, in which the dynamic viscosity is evaluated at $T_{\infty}$. The transformed boundary conditions are

$$
\begin{aligned}
f(\xi, 0)=0, & \theta(\xi, 0)=1, \\
f^{\prime}(\xi, \infty)=1, & \theta(\xi, \infty)=0 .
\end{aligned}
$$

The physical quantities of major interest are the velocity components $u$ and $v$, and the local nusselt number

$$
\begin{gathered}
u(x, y)=u_{\infty} f^{\prime}(\xi, \eta), \\
v(x, y)=-\frac{\alpha \operatorname{Pe}_{x}^{1 / 2}}{2 x}\left((m+1) f(\xi, \eta)+(m-1) \eta f^{\prime}+(2 \lambda-3 m-1) \xi \frac{\partial f(\xi, \eta)}{\partial \xi}\right), \\
\frac{N u_{x}}{\operatorname{Pe}_{x}^{1 / 2}}=-\theta^{\prime}(\xi, 0) .
\end{gathered}
$$




\subsection{The Disturbance Flow}

In the usual manner for linear stability analysis, the velocities, pressure, temperature, and viscosity are assumed to be the sum of mean and fluctuating components, here denoted as subscripts 0 and 1 quantities, respectively,

$$
\begin{aligned}
u(x, y, z, t) & =u_{0}(x, y)+u_{1}(x, y, z, t), \\
v(x, y, z, t) & =v_{0}(x, y)+v_{1}(x, y, z, t), \\
w(x, y, z, t) & =w_{1}(x, y, z, t), \\
P(x, y, z, t) & =P_{0}(x, y)+P_{1}(x, y, z, t), \\
T(x, y, z, t) & =T_{0}(x, y)+T_{1}(x, y, z, t), \\
\mu(T) & =\mu_{0}\left(T_{0}\right)+\mu_{1}\left(T_{1}\right) .
\end{aligned}
$$

After substituting (2.15) into the governing equations for the three dimensional convective flow in a porous medium, the base flow quantities are subtracted, with the terms higher than first order in disturbance quantities being neglected. Then we get the following disturbance equations:

$$
\begin{gathered}
\frac{\partial u_{1}}{\partial x}+\frac{\partial v_{1}}{\partial y}+\frac{\partial w_{1}}{\partial z}=0 \\
\mu_{0} u_{1}+\mu_{1} u_{0}+2 K^{*} \rho_{\infty} u_{0} u_{1}=-K \frac{\partial P_{1}}{\partial x} \\
\mu_{0} v_{1}+\mu_{1} v_{0}+2 K^{*} \rho_{\infty} v_{0} v_{1}=-K\left(\frac{\partial P_{1}}{\partial y}-\rho_{\infty} g \beta T_{1}\right), \\
\mu_{0} w_{1}=-K \frac{\partial P_{1}}{\partial z} \\
u_{0} \frac{\partial T_{1}}{\partial x}+v_{0} \frac{\partial T_{1}}{\partial y}+u_{1} \frac{\partial T_{0}}{\partial x}+v_{1} \frac{\partial T_{0}}{\partial y}=\alpha\left[\frac{\partial^{2} T_{1}}{\partial x^{2}}+\frac{\partial^{2} T_{1}}{\partial y^{2}}+\frac{\partial^{2} T_{1}}{\partial z^{2}}\right] .
\end{gathered}
$$

Following the method of order of magnitude analysis described in detail by Hsu and Cheng [1], the terms $\partial u_{1} / \partial x$ and $\partial^{2} T_{1} / \partial x^{2}$ in (2.16) and (2.20) can be neglected. The omission of $\partial u_{1} / \partial x$ in (2.16) implies the existence of a disturbance stream function $\Psi_{1}$, such as

$$
w_{1}=\frac{\partial \Psi_{1}}{\partial y}, \quad v_{1}=-\frac{\partial \Psi_{1}}{\partial z}
$$


Eliminating $P_{1}$ from (2.17)-(2.19) with the aid of (2.21) leads to

$$
\begin{gathered}
u_{0} \frac{\partial \mu_{1}}{\partial z}+\left(\mu_{0}+2 K^{*} \rho_{\infty} u_{0}\right) \frac{\partial u_{1}}{\partial z}=\mu_{0} \frac{\partial^{2} \Psi_{1}}{\partial x \partial y}+\frac{\partial \Psi_{1}}{\partial y} \frac{\partial \mu_{0}}{\partial x} \\
-\left(\mu_{0}+2 K^{*} \rho_{\infty} v_{0}\right) \frac{\partial^{2} \Psi_{1}}{\partial z^{2}}+v_{0} \frac{\partial \mu_{1}}{\partial z}=\mu_{0} \frac{\partial^{2} \Psi_{1}}{\partial y^{2}}+\frac{\partial \mu_{0}}{\partial y} \frac{\partial \Psi_{1}}{\partial y}+K \rho_{\infty} g \beta \frac{\partial T_{1}}{\partial z} \\
u_{0} \frac{\partial T_{1}}{\partial x}+v_{0} \frac{\partial T_{1}}{\partial y}+u_{1} \frac{\partial T_{0}}{\partial x}-\frac{\partial \Psi_{1}}{\partial z} \frac{\partial T_{0}}{\partial y}=\alpha\left(\frac{\partial^{2} T_{1}}{\partial y^{2}}+\frac{\partial^{2} T_{1}}{\partial z^{2}}\right) .
\end{gathered}
$$

As in Hsu and Cheng [1], we assume that the three dimensionless disturbances for neutral stability are of the form

$$
\left(\Psi_{1}, u_{1}, T_{1}\right)=[\bar{\Psi}(x, y), \bar{u}(x, y), \bar{T}(x, y)] e^{i a z},
$$

where $a$ is the spanwise periodic wave number. Substituting (2.23) into (2.22) yields

$$
\begin{gathered}
\bar{u}=\left(\frac{1}{1+\left(2 K^{*} / v_{\infty}\right)\left(\mu^{*}\right)^{-\theta} u_{0}}\right)\left[\frac{1}{i a}\left(\frac{\partial^{2} \bar{\Psi}}{\partial x \partial y}+\left(\ln \mu^{*}\right) \frac{\partial \bar{\Psi}}{\partial y} \frac{\partial \theta}{\partial x}\right)-\left(\ln \mu^{*}\right) \frac{u_{0} \bar{T}}{T_{w}-T_{\infty}}\right] \\
\frac{\partial^{2} \bar{\Psi}}{\partial y^{2}}-a^{2}\left(1+\frac{2 K^{*}}{v_{\infty}}\left(\mu^{*}\right)^{-\theta} v_{0}\right) \bar{\Psi}-i a v_{0}\left(\ln \mu^{*}\right) \frac{\bar{T}}{T_{w}-T_{\infty}}+\left(\ln \mu^{*}\right) \frac{\partial \theta}{\partial y} \frac{\partial \bar{\Psi}}{\partial y}=-\frac{i a K \rho_{\infty} g \beta}{\mu_{\infty}}\left(\mu^{*}\right)^{-\theta} \bar{T} \\
u_{0} \frac{\partial \bar{T}}{\partial x}+v_{0} \frac{\partial \bar{T}}{\partial y}+\bar{u} \frac{\partial T_{0}}{\partial x}-i a \bar{\Psi} \frac{\partial T_{0}}{\partial y}=\alpha\left(\frac{\partial^{2} \bar{T}}{\partial y^{2}}-a^{2} \bar{T}\right) .
\end{gathered}
$$

Equation (2.24) is solved based on the local similarity approximation (see [22]), wherein the disturbances are assumed to have weak dependence in the streamwise direction (i.e., $\partial / \partial x \ll \partial / \partial \eta)$. To facilitate the analysis, the following transformations are introduced to nondimensionalize the preceding equations

$$
k=\frac{a x}{\operatorname{Pe}_{x}^{1 / 2}}, \quad F(\eta)=\frac{\bar{\Psi}}{i \alpha \mathrm{Pe}_{x}^{1 / 2}}, \quad \Theta(\eta)=\frac{\bar{T}}{T_{w}-T_{\infty}} .
$$




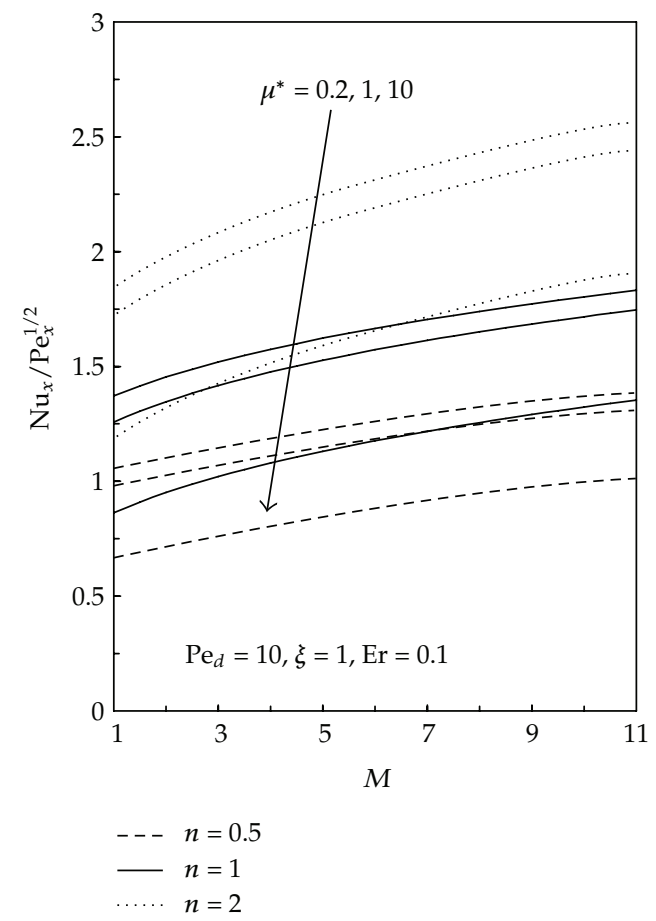

Figure 1: The local Nusselt number as a function of $M$ for selected values of $\mu^{*}$ and $n$ for the case $m=0$.

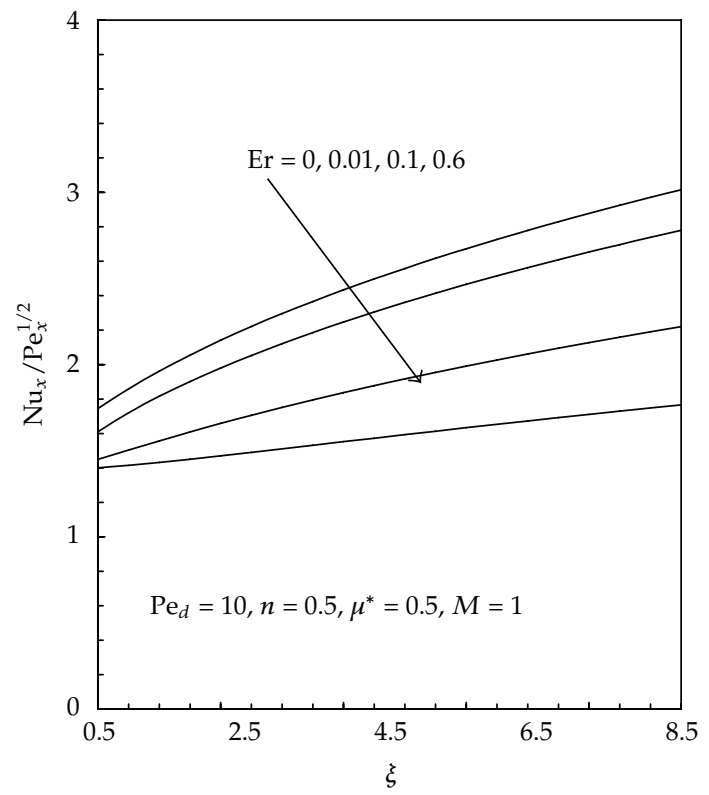

Figure 2: The local Nusselt number as a function of $\xi$ for various values of Er for the case $m=0$. 


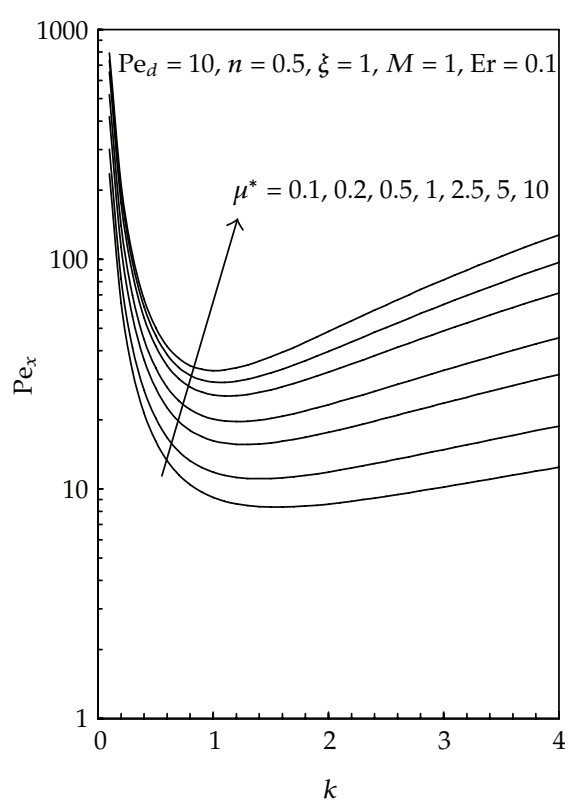

Figure 3: Neutral stability curves for selected values of $\mu^{*}$ for the case $m=0$.

Introducing (2.25) into (2.24) gives

$$
\begin{aligned}
F^{\prime \prime}+ & \left(\ln \mu^{*}\right) \theta^{\prime} F^{\prime}-k^{2}\left[1-\frac{2 \operatorname{ErPe}_{d} \xi^{2 m /(2 \lambda-3 m-1)}\left(\mu^{*}\right)^{-\theta}}{\operatorname{Pe}_{x}^{1 / 2}} H_{1}\right] F+\frac{k\left(\ln \mu^{*}\right)}{\operatorname{Pe}_{x}^{1 / 2}} H_{1} \Theta \\
= & -M k \operatorname{Pe}_{x}^{1 / 2} \xi\left(\mu^{*}\right)^{-\theta} \Theta, \\
\Theta^{\prime \prime}+ & H_{3} \Theta^{\prime}-\left[k^{2}+\lambda f^{\prime}-\frac{\left(\ln \mu^{*}\right) f^{\prime} H_{2}}{1+2 \operatorname{ErPe}_{d} \xi^{2 m /(2 \lambda-3 m-1)}\left(\mu^{*}\right)^{-\theta} f^{\prime}}\right] \Theta \\
& -\frac{H_{2}\left[((m-1) / 2) \eta F^{\prime \prime}+\left(m+\ln \mu^{*} H_{4}\right) F^{\prime}\right]}{k \operatorname{Pe}_{x}^{1 / 2}\left(1+2 \operatorname{ErPe}_{d} \xi^{2 m /(2 \lambda-3 m-1)}\left(\mu^{*}\right)^{-\theta} f^{\prime}\right)}=k \operatorname{Pe}_{x}^{1 / 2} \theta^{\prime} F,
\end{aligned}
$$

subject to the boundary conditions

$$
F(0)=F(\infty)=\Theta(0)=\Theta(\infty)=0,
$$




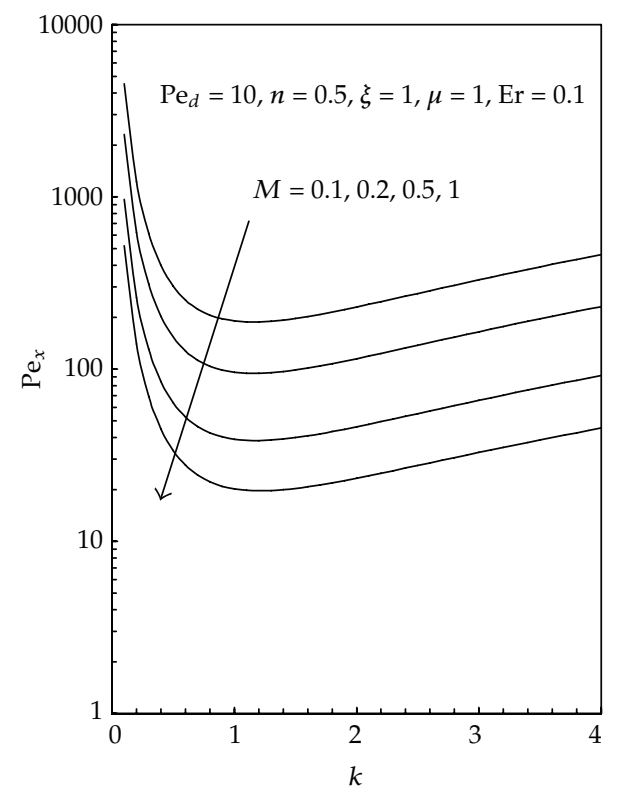

Figure 4: Neutral stability curves for selected values of $M$ for the case $m=0$.

where the coefficients $H_{1}-H_{4}$ are given by

$$
\begin{aligned}
& H_{1}=\frac{m+1}{2} f+\frac{m-1}{2} \eta f^{\prime}+\frac{2 \lambda-3 m-1}{2} \xi \frac{\partial f}{\partial \xi^{\prime}}, \\
& H_{2}=\lambda \theta+\frac{m-1}{2} \eta \theta^{\prime}+\frac{2 \lambda-3 m-1}{2} \xi \frac{\partial \theta}{\partial \xi^{\prime}} \\
& H_{3}=\frac{m+1}{2} f+\frac{2 \lambda-3 m-1}{2} \xi \frac{\partial f}{\partial \xi^{\prime}} \\
& H_{4}=\frac{m-1}{2} \eta \theta^{\prime}+\frac{2 \lambda-3 m-1}{2} \xi \frac{\partial \theta}{\partial \xi^{\prime}}
\end{aligned}
$$

Equations (2.26) and (2.27) constitute a second-order system of linear ordinary differential equations for the disturbance amplitude distributions $F(\eta)$ and $\Theta(\eta)$. For fixed values of $\xi, \lambda, m, M, \mathrm{Er}, \mathrm{Pe}_{d}, k$, and $\mu^{*}$, the solution $F$ and $\Theta$ is an eigenfunction for the eigenvalue $\mathrm{Pe}_{x}$. We note that (2.26) and (2.27) under boundary conditions (2.28) for $\mu^{*}=1, \operatorname{Er}=0, \xi=1$, and $\partial / \partial \xi=0$ are reduced to those given in Hsu and Cheng [1], where the Darcy model with constant viscosity is considered.

\section{Numerical Scheme}

In this section, we compute the approximate value of $\mathrm{Pe}_{x}$ for (2.26) and (2.27) with the boundary conditions (2.28). An implicit finite difference method is used to solve first the base flow (2.11) and (2.12) with the boundary conditions (2.13), and the results are stored for a fixed step size $h$, which is small enough to predict accurate linear interpolation between 


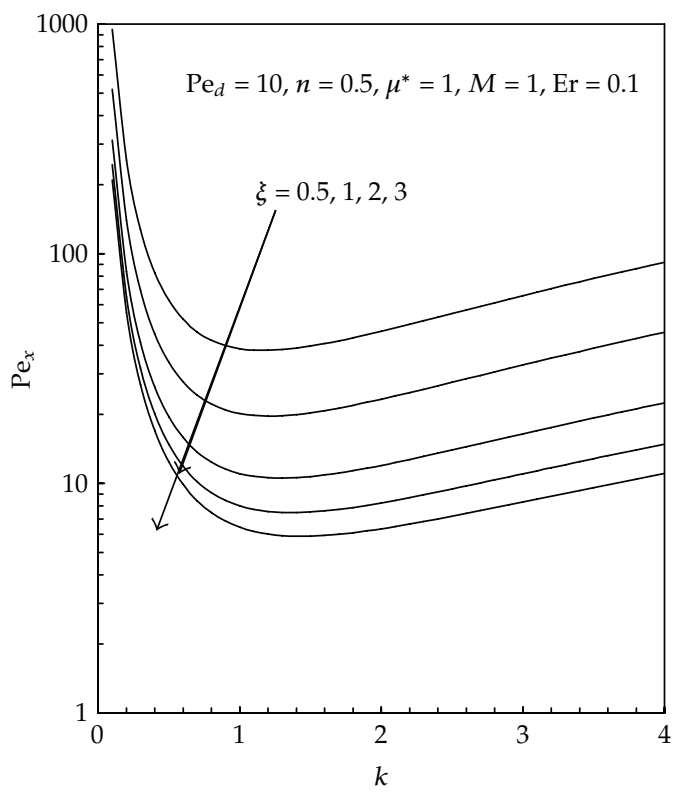

Figure 5: Neutral stability curves for selected values of $\xi$ for the case $m=0$.

mesh point. The domain is $0 \leq \eta \leq \eta_{\infty}$, where $\eta_{\infty}$ is the edge of the boundary layer of the basic flow. For a positive integer $N$, let $h=\eta_{\infty} / N$ and $\eta_{i}=i h, i=0,1, \ldots, N$, the problem is discretized with standard centered finite differences of order 2, following Usmani [23]. Solving eigenvalue problem is achieved by using the subroutine GVLRG of the IMSL library Inc., see [24].

\section{Results and Discussion}

Numerical results for the local Nusselt number, neutral stability curves, the critical Peclet, and associate wave numbers at the onset of vortex instability are presented for a range of wall to ambient viscosity ratio parameter $\mu^{*}$ from 0.1 to 10 . As the temperature is increased, the gas viscosity increases, while the liquid viscosity decreases [20]. Therefore, for a heated wall, values of $\mu^{*}>1$ correspond to the case of gas heating, and values of $\mu^{*}<1$ corresponds to the case of liquid heating. The effect of the nonuniform temperature profile on the wall is also studied, and corresponds to variations of the parameter $\lambda$. Because of lack of space, we shall only outline the numerical results of the case $m=0$ (assisting flow over a horizontal flat plate at zero incident) and results of the case $m=1$ (stagnation point flow about a horizontal surface) can be omitted.

The local Nusselt number as functions of $M$ and $\xi$ for various values of $\mu^{*}, n$, and Er are shown, respectively, in Figures 1 and 2. It is seen that higher Nusselt number occurs at higher values of $M, n, \xi$, and lower values of $\mathrm{Er}, \mu^{*}$.

Figures 3, 4, and 5, respectively, show the neutral stability curves, in terms of the Peclet number $\mathrm{Pe}_{x}$ and the dimensionless wave number $k$ for selected values of $\mu^{*}, M$, and $\xi$. It is observed that, as $\mu^{*}$ increases, the neutral stability curves shift to higher Peclet number, while as $\xi$ and $M$ increase, the neutral stability curves shift to lower Peclet number $\mathrm{Pe}_{x}$. 


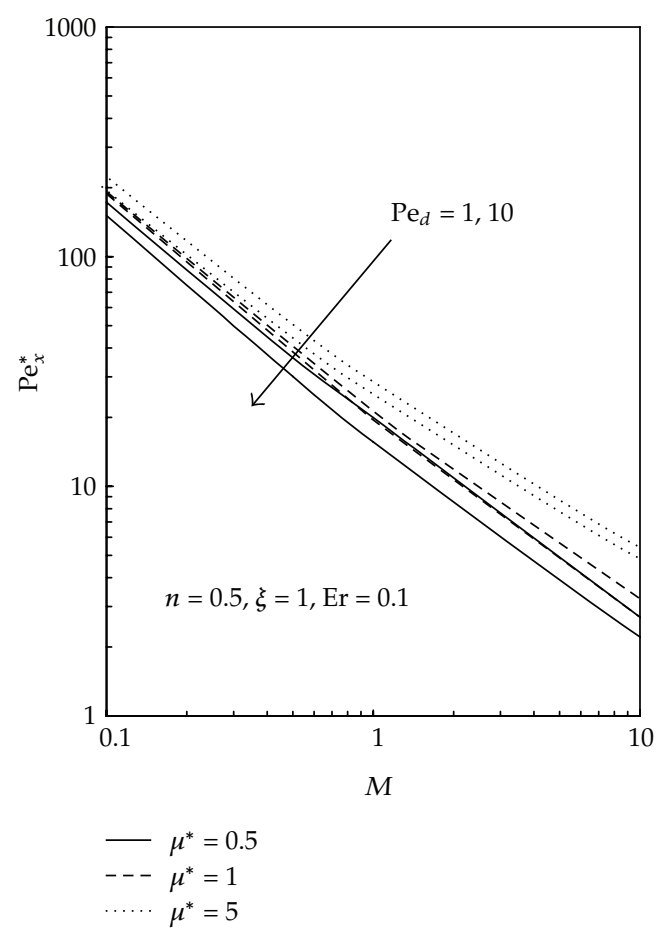

Figure 6: Critical Peclet numbers as a function of $M$ for selected values of $\mathrm{Pe}_{d}$ and $\mu^{*}$ for the case $m=0$.

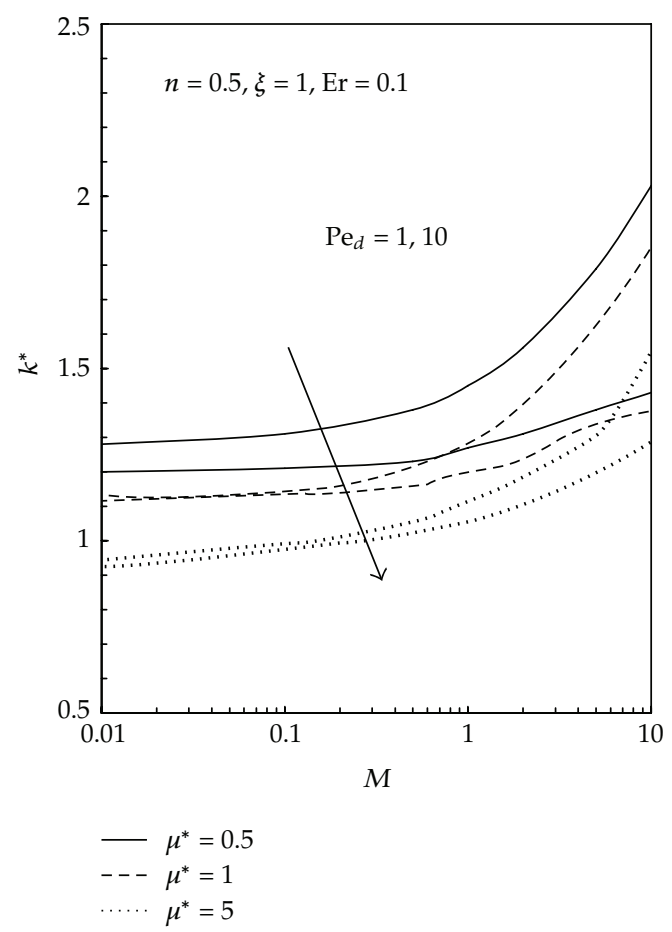

Figure 7: Critical wave numbers as a function of $M$ for the selected values of $\operatorname{Pe}_{d}$ and $\mu^{*}$ for the case $m=0$. 
The critical Peclet number and wave number are plotted as a function of $M$ for selected values of $\mu^{*}$ and $\mathrm{Pe}_{d}$ in Figures 6 and 7. It can be seen that as the variable viscosity parameter $\mu^{*}$ increases, the critical Peclet number $\mathrm{Pe}_{x}^{*}$ increases, while as $\mathrm{Pe}_{d}$ or $M$ increases, the critical Peclet number $\mathrm{Pe}_{x}^{*}$ decreases. Further, higher critical wave number occurs at higher values of $M$, and lower values of $\mu^{*}$ or $\mathrm{Pe}_{d}$.

Finally, we conclude that the variable viscosity effect enhances the heat transfer rate and destabilizes the flow for liquid heating while the opposite tend is true for gas heating.

\section{Conclusions}

The non-Darcy mixed convection flow on a semi-infinite, nonisothermal horizontal plate embedded in a porous medium with variable viscosity, is investigated. The non-Darcy model, which includes the Ergun extension, is employed to describe the base and disturbed flows in the porous medium. The variation of viscosity is expressed as an exponential function of temperature. The effects of variable viscosity characterized by the parameter $\mu^{*}$ on the flow and vortex instability are examined. The surface temperature is assumed to vary as a power function of the distance from the origin. The governing partial differential equations are transformed to a nonsimilar form by introducing appropriate transformations and are solved numerically using an implicit finite difference scheme. The resulting eigenvalue problem is solved by using a finite difference scheme. The effects of all involved parameters on the local Nusselt number, critical Peclet and associated wave number are presented. It is shown that, for liquid heating, the variable viscosity effect enhances the heat transfer rate and destabilizes flow, while, for gas heating, the opposite trend is true.

\section{Nomenclature}

a: $\quad$ Spanwise wave number

$d$ : $\quad$ Mean particle diameter or pore diameter

$f$ : Dimensionless base state stream function

$F: \quad$ Dimensionless disturbance stream function

g: $\quad$ Gravitational acceleration

$i$ : Complex number

$k$ : $\quad$ Dimensionless wave number

$K: \quad$ Permeability of porous medium

$K^{*}: \quad$ Inertial coefficient in Ergun Equation

$\mathrm{Nu}_{x}: \quad$ Local Nusselt number

$P: \quad$ Pressure

$\mathrm{Pe}_{x}$ : Local Peclet number

$\mathrm{Pe}_{d}$ : Peclet number based on the pore diameter

$T: \quad$ Fluid temperature

$u, v, w$ : Volume-averaged velocity in the $x, y$, and $z$ directions

$x, y, z$ : Axial, normal, and spanwise coordinates. 


\title{
Greek Symbols
}

\author{
$\alpha$ : Thermal diffusivity \\ $\beta$ : Volumetric coefficient of thermal expansion \\ $\eta$ : Pseudosimilarity variable \\ $\theta$ : Dimensionless base state temperature \\ $\Theta$ : Dimensionless disturbance temperature \\ $\mu$ : Dynamic viscosity of the fluid \\ $\xi$ : Nonsimilarity parameter \\ $\lambda$ : Exponent in the wall temperature variation \\ $v$ : Kinematic viscosity \\ $\mu^{*}$ : Wall to ambient viscosity ratio, $\mu^{*}=\mu_{w} / \mu_{\infty}$ \\ $\psi$ : Stream function
}

\section{Subscripts}

$w$ : Conditions at the wall

$\infty$ : Conditions at the free stream

0: Basic undisturbed quantities

1: Disturbed quantities

\section{Superscripts}

*: Critical value

': Differentiation with respect to $\eta$.

\section{References}

[1] C. T. Hsu and P. Cheng, "Vortex instability of mixed convective flow in a semi-infinite porous medium bounded by a horizontal surface," International Journal of Heat and Mass Transfer, vol. 23, no. 6, pp. 789$798,1980$.

[2] J. Jiin-Yuh and C. Wen-Jeng, "Vortex instability of buoyancy-induced inclined boundary layer flow in a saturated porous medium," International Journal of Heat and Mass Transfer, vol. 31, no. 4, pp. 759-767, 1988.

[3] J. Jiin-Yuh and L. Kun-Nun, "Vortex instability of mixed convection flow over horizontal and inclined surfaces in a porous medium," International Journal of Heat and Mass Transfer, vol. 35, no. 9, pp. 20772085, 1992.

[4] I. A. Hassanien, A. A. Salama, and A. M. Elaiw, "Variable permeability effect on vortex instability of mixed convection flow in a semi-infinite porous medium bounded by a horizontal surface," Applied Mathematics and Computation, vol. 146, no. 2-3, pp. 829-847, 2003.

[5] A. M. Elaiw, "Vortex instability of mixed convection boundary layer flow adjacent to a non-isothermal inclined surface in a porous medium with variable permeability," Zeitschrift für Angewandte Mathematik und Mechanik, vol. 88, no. 2, pp. 121-128, 2008.

[6] A. M. Elaiw and F. S. Ibrahim, "Vortex instability of mixed convection boundary layer flow adjacent to a nonisothermal horizontal surface in a porous medium with variable permeability," Journal of Porous Media, vol. 11, no. 3, pp. 305-321, 2008.

[7] A. M. Elaiw, F. S. Ibrahim, and A. A. Bakr, "The influence of variable permeability on vortex instability of a horizontal combined free and mixed convection flow in a saturated porous medium," Zeitschrift für Angewandte Mathematik und Mechanik, vol. 87, no. 7, pp. 528-536, 2007.

[8] J. Y. Jang and J. L. Chen, "Thermal dispersion and inertia effects on vortex instability of a horizontal mixed convection flow in a saturated porous medium," International Journal of Heat and Mass Transfer, vol. 36, no. 2, pp. 383-389, 1993. 
[9] D. H. Lee, D. Y. Yoon, and C. K. Choi, "The onset of vortex instability in laminar natural convection flow over an inclined plate embedded in a porous medium," International Journal of Heat and Mass Transfer, vol. 43, no. 16, pp. 2895-2908, 2000.

[10] T. J. Chung, J. H. Park, C. K. Choi, and D. Y. Yoon, "The onset of vortex instability in laminar forced convection flow through a horizontal porous channel," International Journal of Heat and Mass Transfer, vol. 45, no. 14, pp. 3061-3064, 2002.

[11] D. A. Nield and A. Bejan, Convection in Porous Media, Springer, New York, NY, USA, 3rd edition, 2006.

[12] D. R. Kassoy and A. Zebib, "Variable viscosity effects on the onset of convection in porous media," Physics of Fluids, vol. 18, no. 12, pp. 1649-1651, 1975.

[13] J. Gary, D. R. Kassoy, H. Tadjeran, and A. Zebib, "The effect of significant viscosity variation on convective heat transport in water saturated porous media," Journal of Fluid Mechanics, vol. 117, pp. 233-249, 1982.

[14] J. X. Ling and A. Dybbs, "Effect of variable viscosity on forced convection over a flat plate submersed in a porous medium," Journal of Heat Transfer, vol. 114, no. 4, pp. 1063-1065, 1992.

[15] F. C. Lai and F. A. Kulacki, "The effect of variable viscosity on convective heat transfer along a vertical surface in a saturated porous medium," International Journal of Heat and Mass Transfer, vol. 33, no. 5, pp. 1028-1031, 1990.

[16] M. Kumari, "Effect of variable viscosity on non-Darcy free or mixed convection flow on a horizontal surface in a saturated porous medium," International Communications in Heat and Mass Transfer, vol. 28, no. 5, pp. 723-732, 2001.

[17] I. A. Hassanien, A. H. Essawy, and N. M. Moursy, "Variable viscosity and thermal conductivity effects on combined heat and mass transfer in mixed convection over a UHF/UMF wedge in porous media: the entire regime," Applied Mathematics and Computation, vol. 145, no. 2-3, pp. 667-682, 2003.

[18] K. E. Chin, R. Nazar, N. M. Arifin, and I. Pop, "Effect of variable viscosity on mixed convection boundary layer flow over a vertical surface embedded in a porous medium," International Communications in Heat and Mass Transfer, vol. 34, no. 4, pp. 464-473, 2007.

[19] M. A. Seddeek, "Effects of magnetic field and variable viscosity on forced non-Darcy flow about a flat plate with variable wall temperature in porous media in the presence of suction and blowing," Journal of Applied Mechanics and Technical Physics, vol. 43, pp. 13-17, 2002.

[20] J. Y. Jang and J. S. Leu, "Variable viscosity effects on the vortex instability of free convection boundary layer flow over a horizontal surface in a porous medium," International Journal of Heat and Mass Transfer, vol. 36, no. 5, pp. 1287-1294, 1993.

[21] S. Ergun, "Fluid flow through packed columns," Chemical Engineering Science, vol. 48, pp. 89-94, 1952.

[22] E.M. Sparrow, H. Quack, and C.T. Boerner, "Local nonsimilarity boundary layer solutions," AIAA Journal, vol. 8, pp. 1936-1942, 1970.

[23] R. A. Usmani, "Some new finite difference methods for computing eigenvalues of two-point boundary value problems," Computers E Mathematics with Applications, vol. 11, no. 9, pp. 903-909, 1985.

[24] IMSL, References Manual, IMSL, Houston, Tex, 1990. 


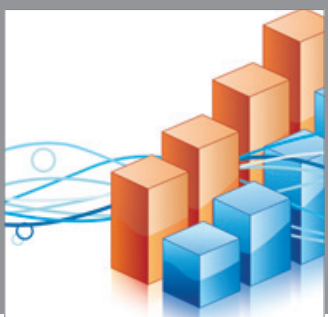

Advances in

Operations Research

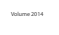

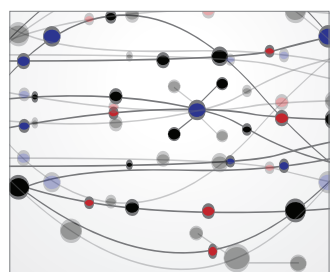

\section{The Scientific} World Journal
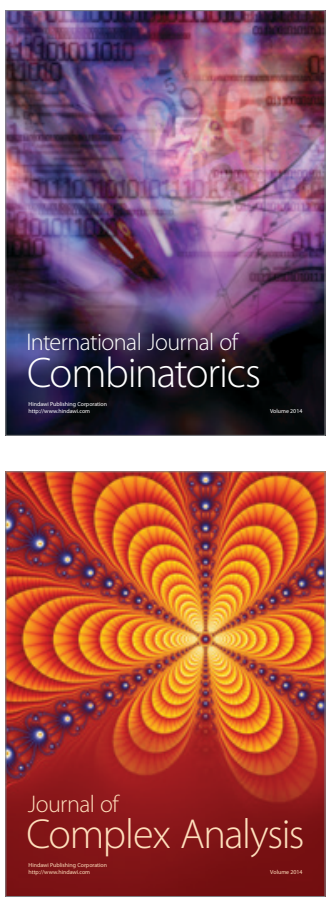

International Journal of

Mathematics and

Mathematical

Sciences
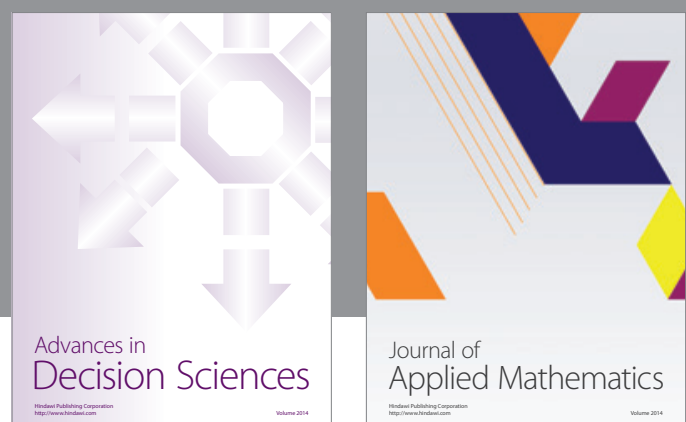

Journal of

Applied Mathematics
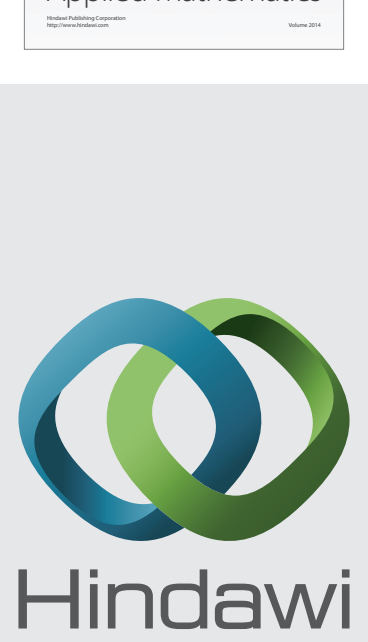

Submit your manuscripts at http://www.hindawi.com
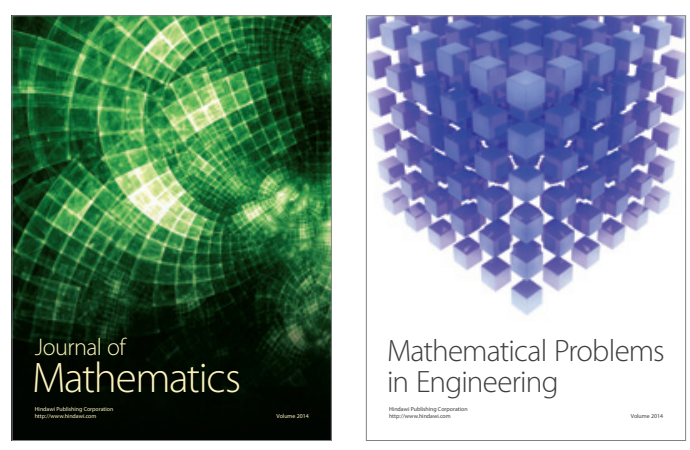

Mathematical Problems in Engineering
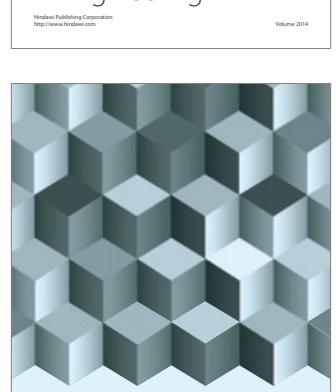

Journal of

Function Spaces
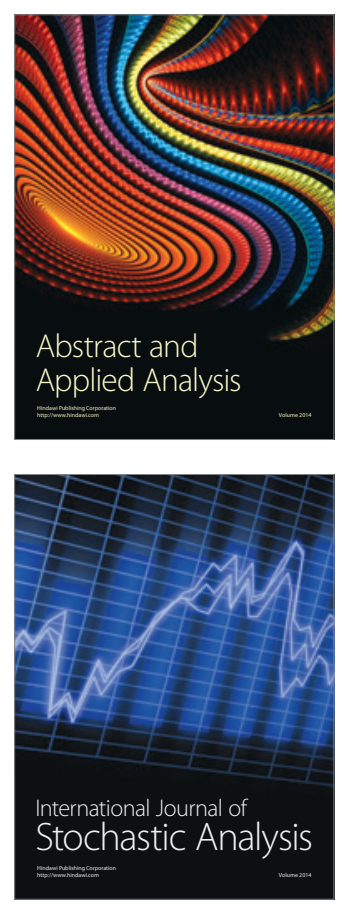

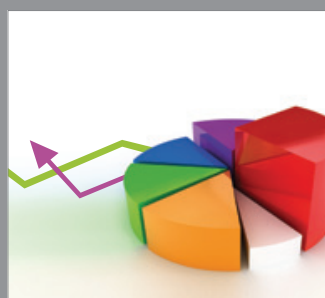

ournal of

Probability and Statistics

Promensencen
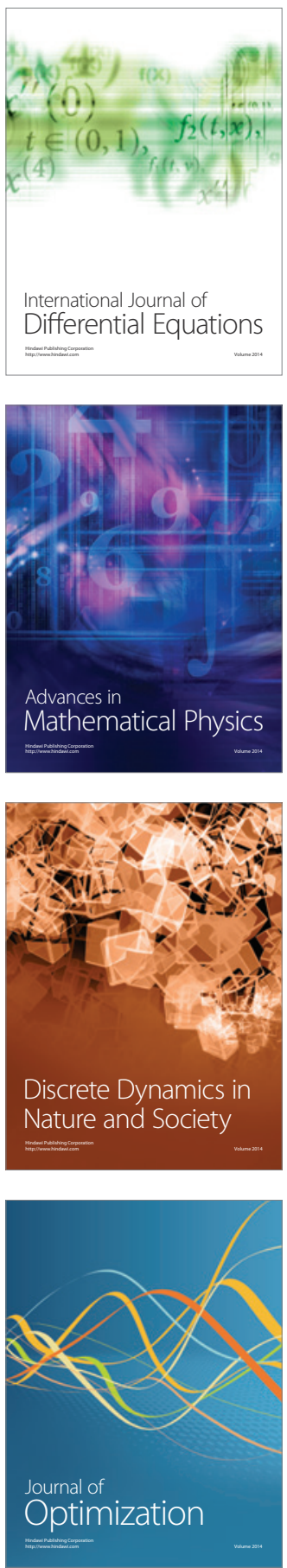